\title{
Conduit Puncture for Electrophysiological Procedures in Patients with Fontan Circulation
}

Jae-Sun Uhm¹, Nam Kyun Kim², Boyoung Joung ${ }^{1}$, Hui-Nam Pak ${ }^{1}$, Moon-Hyoung Lee

Departments of Cardiology ${ }^{1}$ and Pediatric Cardiology ${ }^{2}$, Severance Cardiovascular Hospital, Yonsei University College of Medicine, Seoul, Korea
Received: August 23, 2015

Revision Received: November 1, 2015 Accepted: December 15, 2015

Correspondence: Jae-Sun Uhm, MD, PhD

Department of Cardiology, Severance Cardiovascular Hospital, Yonsei University College of Medicine, 50-1 Yonsei-ro Seodaemun-gu, Seoul, Republic of Korea, 03722

Tel: +82-2-2228-8441, Fax: +82-2-2227-7732

E-mail: jason@yuhs.ac

Copyright $(2015$ The Official Journal of Korean Heart Rhythm Society Editorial Board \& MMK Co., Ltd.

\begin{abstract}
Background: Electrophysiological procedures are challenging in patients who have undergone lateral tunnel or extracardiac conduit Fontan operation because the caval veins are not connected to the cardiac atria and ventricles. This study describes our experience in managing a series of patients with Fontan circulation requiring catheter ablation for arrhythmias.

Methods: This study included eight consecutive patients with Fontan circulation who underwent catheter ablation or pacemaker implantation via Fontan conduit puncture [median age (interquartile range), 21.5 (16.0-25.8) years; 5 men]. Lateral tunnel and extracardiac conduit were equally distributed among the eight patients. A standard technique for conduit puncture and subsequent electrophysiologic procedure was used. The time taken for conduit puncture was compared for different types of conduits.
\end{abstract}

Results: The median age of patients in this series was 21.5 years (interquartile range: 16.0-25.8 years). Fontan conduit puncture via right femoral vein under intracardiac echocardiographic guidance was successfully performed without complications in seven of the eight patients. Conduit puncture failed in one patient with extracardiac conduit made of the pericardium due to interruption of both femoral veins. In three patients with Fontan conduit made of pericardium, a Bronckenbrough transseptal needle or a radiofrequency transseptal needle with a snare was used. In four patients with Fontan conduit made of Gore-tex, a radiofrequency transseptal needle with a snare, and percutaneous transluminal angioplasty balloon were used. Fontan conduit puncture time was significantly longer in patients with conduit made of Gore-tex (median time, $91 \mathrm{~min}$; interquartile range, 59.8-130.5 $\mathrm{min}$ ) than in patients with conduit made of the pericardium (median time, $11.5 \mathrm{~min}$; interquartile range, 10.0-18.3 $\min )$, respectively $(p=0.020)$.

Conclusions: Conduit puncture is feasible and safe in patients with lateral tunnel and extracardiac Fontan circulation. Puncture of the Gore-tex conduit is more difficult and time consuming than puncture of the pericardium conduit.

Key Words: - Catheter Ablation - Fontan Operation - Conduit Puncture -Transseptal Needle 


\section{Introduction}

Survival of patients with complex congenital heart disease has increased in recent years due to improvements in surgical and interventional techniques as well as in medical management. ${ }^{1}$ As a result, there is an increase in the number of adult patients with congenital heart disease. Since arrhythmias and heart failure are common issues in adult patients with congenital heart disease, it is not surprising that there is a rise in the demand for electro physiological studies and cardiac implantable electronic device implantations in these patients. ${ }^{1}$

Fontan operation is a standard palliative procedure for functionally single ventricle. ${ }^{2}$ First-generation Fontan operation involves direct connection of the right atrium to the pulmonary artery. ${ }^{3}$ Second and third-generation Fontan operations create a total cavopulmonary connection by using either the lateral tunnel (an intra-atrial tunnel) or the extracardiac conduit (Figure 1). ${ }^{4}$ The conduit is usually made of autologous pericardium or Goretex.

Electrophysiological procedures are challenging in patients who have undergone the lateral tunnel or extracardiac conduit Fontan operation because the caval veins are not connected to the atria and ventricles. The present study is performed to demonstrate feasibility and safety of conduit puncture in patients who have undergone lateral tunnel or extracardiac conduit Fontan operation.

\section{Methods}

This study included all consecutive patients in the period between December 2013 to February 2015 requiring catheter ablation or pacemaker implantation for arrhythmias after Fontan operation. Patients had either lateral tunnel or extracardiac conduit Fontan operations using autologous pericardium or Goretex as the conduit material.

All electrophysiological studies were performed under local anesthesia with mild to moderate sedation and required patients to be fasting. Conduit angiography was performed as an initial step in all cases. Before conduit puncture, two quadripolar electrophysiologic catheters were introduced transcutaneously through the femoral artery and the vein respectively. The first catheter was introduced through the femoral artery and passed along the aorta into the ventricle for recording ventricular signals and pacing the ventricle. In patients with lateral tunnel, the second catheter was introduced through the femoral vein, passed through the inferior vena cava and placed in the lateral tunnel at the level of the atrium for recording atrial signals and pacing the atrium. Similarly, in patients with extracardiac conduit, the second catheter was placed in the esophagus at the level of the atrium. for recording atrial signals and pacing the atrium. When ECG showed sinus rhythm at the beginning of an electrophysiological study, basic electrophysiological studies and electric with or without pharmacologic stimulation was performed for tachycardia induction. After tachycardia was induced, conduit puncture was performed.

Conduit puncture was first attempted with a Brockenbrough transseptal needle (BRK-1; St. Jude Medical, Minnetonka, MN, USA) and a Swartz introducer (SR-0 or SL-1; St. Jude Medical) in all patients under intracardiac echocardiography guidance. The dilator tip of the Swartz introducer was held with a snare catheter (PFM Medical, Nonnweiler, Germany) to prevent it from sliding up along the conduit wall (Figure 2). In cases where a
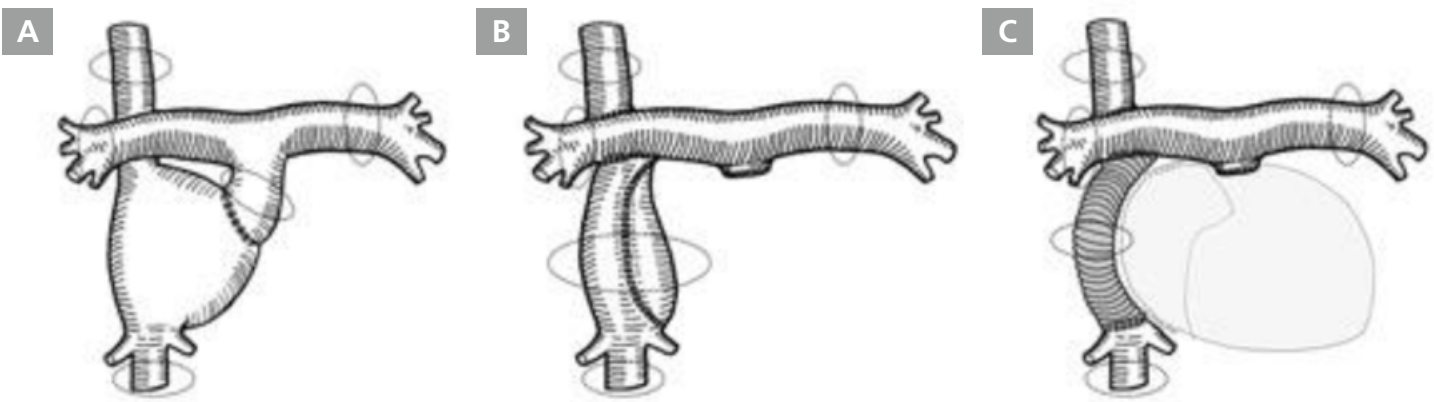

Figure 1. (A) Atriopulmonary connection Fontan operation. (B) Lateral tunnel Fontan operation. (C) Extracardiac conduit Fontan operation. 

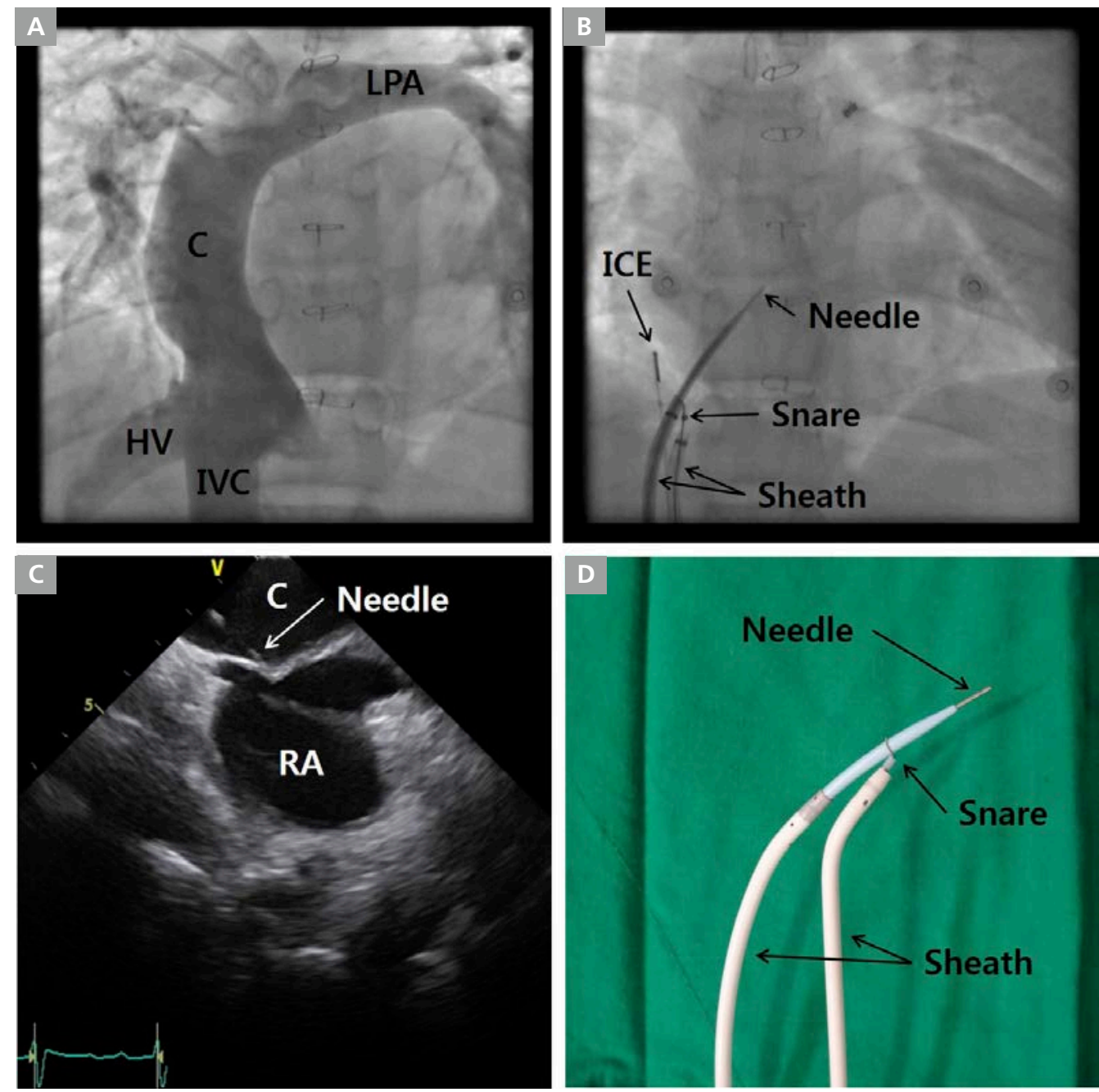

Figure 2. Conduit puncture in lateral tunnel Fontan with pericardium conduit. (A) Fontan conduit angiography. (B) Conduit puncture fluoroscopy. (C) Intracardiac echocardiography during conduit puncture. (D) The Brockenbrough transseptal needle and the Swartz introducer with snare holding the dilator tip as a maneuver to prevent needle sliding up along the conduit wall. C, Fontan conduit; HV, hepatic vein; ICE, intracardiac echocardiography; IVC, inferior vena cava; LPA, left pulmonary artery; RA right atrium.

Brockenbrough transseptal needle failed to create a conduit puncture, a radiofrequency transseptal needle (Baylis Medical Company, Montreal, Canada) was used. Further, if the Swartz introducer could not be inserted into the atrium via the conduit puncture, the conduit puncture was dilated by various sizes and kinds of percutaneous transluminal angioplasty (PTA) balloons inserted over a guidewire into the atrium (Figure 3). Cutting balloons or non-compliant balloons were used where conduit punctures could not be dilated with PTA balloons, failing which dilator of an Inoue balloon (Toray Corporation, Tokyo, Japan) was used (Figure 4).
A conventional mapping maneuver and 3-dimensional electroanatomical mapping with CARTO (Biosense Webster, Diamond Bar, CA, USA) or EnSite NavX (St. Jude Medical) was performed to determine the origin and mechanism of tachycardia. During the procedure, activated clotting time was maintained between 300 to 400 seconds by heparin infusion. Radiofrequency catheter ablation was performed at the point of the earliest electric activity in cases of focal tachycardia or the critical point of the reentry circuit in cases of reentrant tachycardia. The endpoint of an electrophysiological study was termination of tachycardia during radiofrequency energy delivery and non-inducibility of the tachycardia during atrial burst pacing and isoproterenol infusion. 

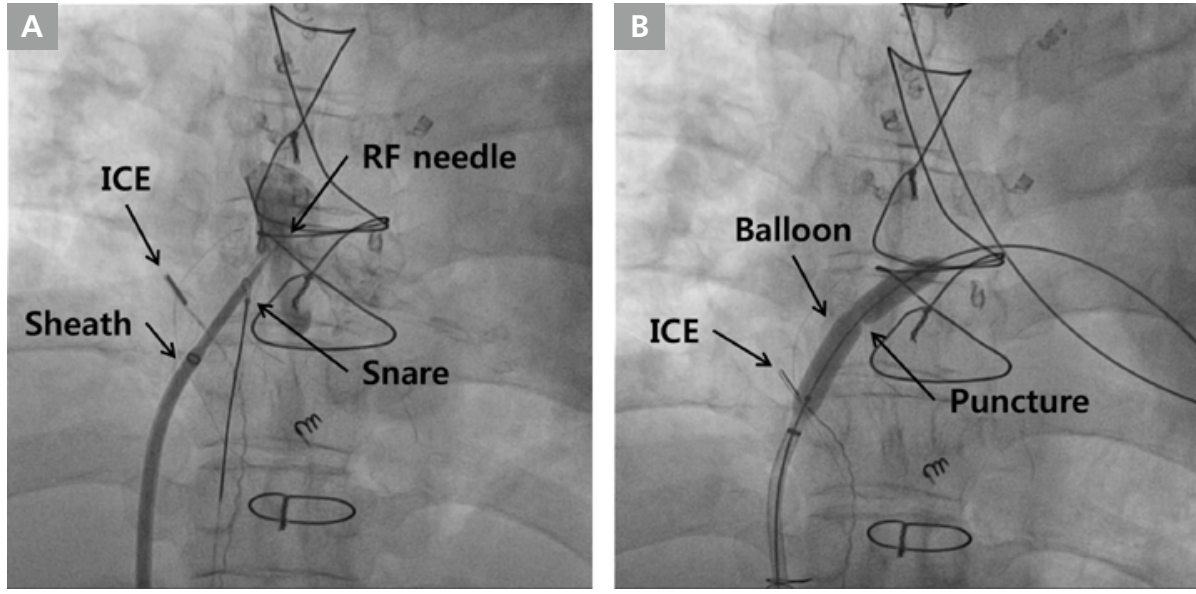

Figure 3. Conduit puncture in extracardiac conduit made of Gore-tex.5 (A) Fluoroscopic image of conduit puncture with radiofrequency transseptal needle stabilized by a snare holding the dilator tip. (B) Fluoroscopic image of dilation of the conduit puncture with a percutaneous transluminal angioplasty balloon.

ICE, intracardiac echocardiography; RF, radiofrequency.
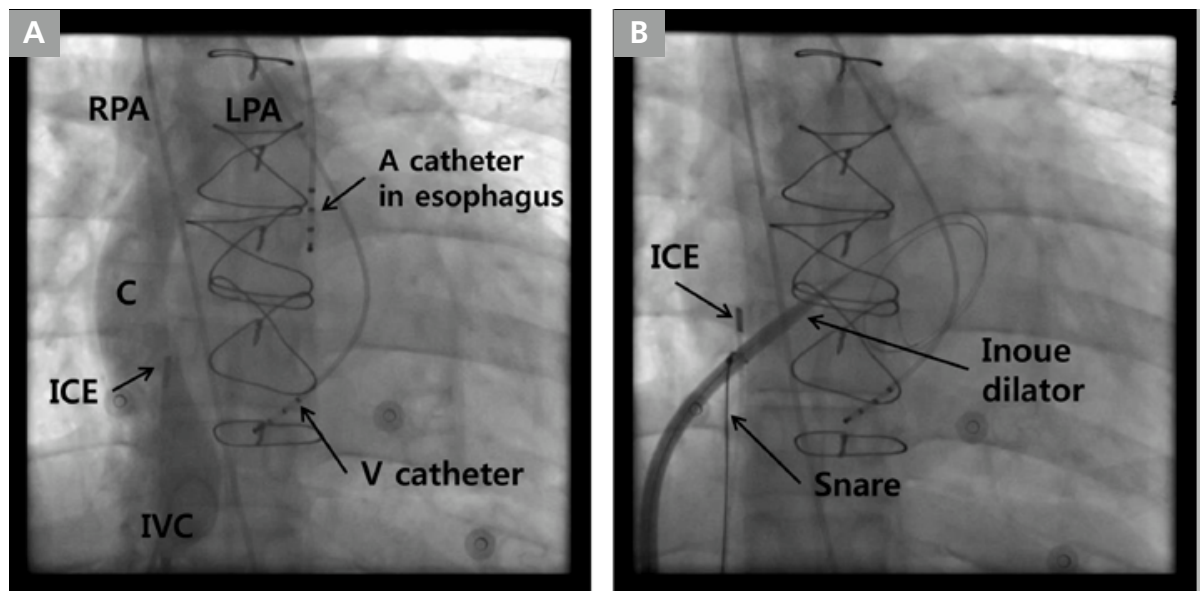

Figure 4. Conduit puncture in extracardiac conduit made of Gore-tex. (A) Fontan conduit angiography. (B) Fluoroscopic image of dilation of the conduit puncture with dilator of an Inoue balloon.

A, atrial; C, Fontan conduit; ICE, intracardiac echocardiography; IVC, inferior vena cava; LPA, left pulmonary artery; RPA, right pulmonary artery; $\mathrm{V}$, ventricular.

The angle between Fontan conduit wall and the inferior vena cava was measured. Time for conduit puncture was recorded. Major complications associated with conduit puncture were defined as hemopericardium or cardiac tamponade, stroke and vascular access site bleeding requiring transfusion.

\section{Statistical Analysis}

Continuous data is presented as median (interquartile range). Puncture times for different conduit materials were compared by using Mann-Whitney test. P-values less than 0.05 were considered statistically significant. Data was analyzed by using the Statistical Package for the Social Sciences (SPSS), version 20.0 (IBM Inc., Armonk, NY, USA).

\section{Results}

A total of eight patients were included in the study. The median age of patients in this study was 21.5 years (interquartile range, 16.0-25.8 years). Five of the patients were men. Lateral tunnel and extracardiac conduit were equally distributed among the eight patients, with four each having either one of the variants of the 
Fontan operation performed respectively. Among patients with lateral tunnel Fontan, three had autologous pericardium and one had Gore-tex as conduit material. On the other hand, in the extracardiac conduit Fontan group, three had Gore-tex conduits while one had autologous pericardium conduit. The indications for electrophysiological study were narrow QRS tachycardia in five patients and atrial flutter in two patients respectively. One patient received a DDDR pacemaker implantation for sick sinus syndrome (Table 1). ${ }^{5}$ In this patient, epicardial pacemaker implantation has been tried and failed because of severe pericardial adhesion and very thin ventricular wall.

The median duration between Fontan operation and onset of tachycardia was 14.1 years (9.5-17.3 years). In seven of eight patients, Fontan conduit puncture could be performed successfully via the right femoral vein and without any complications. In one patient with extracardiac conduit made of autologous pericardium, conduit puncture was unsuccessful due to interruption of bilateral femoral veins. In this case, we tried to puncture the conduit via the right jugular vein, but failed due to an unfavorable angle between the superior vena cava and the conduit wall. Of the remaining three patients with lateral tunnel made of the autologous pericardium, in two a Bronckenbrough transseptal needle and a snare were used and in one patient, a radiofrequency transseptal needle and a snare were used (Figure 2). Among the three patients with extracardiac conduit made of Gore-tex, in two a radiofrequency transseptal needle, a snare, and PTA balloons were used (Figure 3), while in one patient, a radiofrequency transseptal needle, a snare, PTA balloons, cutting balloons, non-compliant balloons, and a dilator for an Inoue balloon were used (Figure 4). In 1 patient among these 2 patients, Fontan conduit was calcified, but puncture was performed without difficulty. In one patient with the lateral tunnel made of Gore-tex, a radiofrequency transseptal needle, a snare, PTA balloons and non-compliant balloons were used. Conduit

Table 1. Baseline characteristics of patients and electrophysiological procedures performed

\begin{tabular}{|c|c|c|c|c|c|c|c|c|}
\hline No & $\begin{array}{c}\text { Age } \\
\text { (Years) }\end{array}$ & Sex & Fontan conduit & CHD & $\begin{array}{c}\text { Type of } \\
\text { arrhythmia }\end{array}$ & Puncture tools & $\begin{array}{l}\text { Puncture time } \\
\text { (min) }\end{array}$ & Outcome \\
\hline 1 & 14 & M & LT, pericardium & DILV, TGA & Septal AT & RF needle, snare & 10 & Success \\
\hline 2 & 31 & M & ECC, Gore-tex & DORV, VSD & SSS & $\begin{array}{l}\text { RF needle, snare, } \\
\text { PTA balloons }\end{array}$ & 62 & Success \\
\hline 3 & 16 & $\mathrm{~F}$ & ECC, pericardium & MA, TGA & NCT & RF needle, snare & 10 & Failure to puncture \\
\hline 4 & 16 & $F$ & LT, pericardium & DORV, AVSD & AVNRT & B needle, snare & 20 & Success \\
\hline 5 & 26 & $\mathrm{~F}$ & ECC, Gore-tex & TGA, VSD & Scar-related AFL & $\begin{array}{l}\text { RF needle, snare, } \\
\text { PTA balloons }\end{array}$ & 59 & Success \\
\hline 6 & 25 & M & LT, Gore-tex & Crisscross, TGA & Scar-related AFL & $\begin{array}{l}\text { RF needle, snare, } \\
\text { NC balloons }\end{array}$ & 120 & Success \\
\hline 7 & 19 & M & LT, pericardium & PA with IVS & Conduit AT & B needle, snare & 13 & Success \\
\hline 8 & 24 & M & ECC, Gore-tex & DORV, TA & Atypical AVNRT & $\begin{array}{l}\text { RF needle, snare, } \\
\text { PTA/NC/cutting } \\
\text { balloons, Inoue dilator }\end{array}$ & 134 & Failure to ablate \\
\hline
\end{tabular}

AFL, atrial flutter; AT, atrial tachycardia; AVNRT, atrioventricular nodal reentrant tachycardia; AVSD, atrioventricular septal defect; $B, B$ Brockenbrough; CHD, congenital heart disease; ECC, extracardiac conduit; DILV, double-inlet left ventricle; DORV, double-outlet right ventricle; LT, lateral tunnel; MA, mitral atresia; NC, non-compliant; NCT, narrow complex tachycardia; PA with IVS, pulmonary atresia with intact ventricular septum; PTA, percutaneous transluminal angioplasty; RF, radiofrequency; SSS, sick sinus syndrome; TA, tricuspid atresia; TGA, transposition of the great arteries; VSD, ventricular septal defect. 
puncture time in patients with Gore-tex conduit was 91 minutes (interquartile range, 59.8-130.5 $\mathrm{min}$ ) while the time taken in patients with conduit made of autologous pericardium was 11.5 minutes (interquartile range, 10.0-18.3 min). The difference was statistically significant $(p=0.020)$. There was no significant association between conduit wall angle and puncture time $(p=0.190)$. There was also no significant association between duration from Fontan operation to conduit puncture and puncture time $(p=0.629)$.

Among the seven patients in whom conduit puncture was achieved, six had successful catheter ablation or pacemaker implantation. One patient with sick sinus syndrome had a ventricular lead successfully implanted into the ventricular apex via the conduit puncture and an atrial lead implanted on the conduit wall at the level of the atrium. ${ }^{5}$ In two patients, scarrelated intraatrial reentrant tachycardia was induced and the critical isthmus was successfully ablated. In two patients, focal atrial tachycardia was induced and the origins of tachycardia, at the interatrial septum in one patient and the conduit wall in another, were ablated. The usual type of atrioventricular nodal reentrant tachycardia was induced and the slow pathway was ablated under potential guidance in one patient. Catheter ablation failed in one patient. In this patient, atrioventricular nodal reentrant tachycardia was induced but a slow pathway could not be found in the rightward inferior extension and anteroseptal areas. The slow pathway was presumed to be located in the leftward area but this could not be confirmed as an unfavorable angle between the conduit puncture and the coronary sinus prevented coronary sinus catheterization.

\section{Discussion}

The present study showed that conduit puncture via the right femoral vein is feasible and safe in patients with lateral tunnel or extracardiac conduit Fontan circulation. The study also demonstrated that puncture of the conduit made of Gore-tex was more difficult and time-consuming compared to the puncture of the conduit made of pericardium.

In patients with lateral tunnel or extracardiac conduit Fontan circulation, it is difficult to perform electrophysiological procedures, because the intracardiac chambers are completely excluded from the systemic venous system. There are various routes described for approaching the intracardiac chambers, namely, trans-aortic, trans-conduit, trans-thoracic, sternotomy and trans-apical approaches. ${ }^{6-9}$ Because thoracotomy and sternotomy approaches require surgical procedures under general anesthesia, trans-conduit and trans-aortic approach are more commonly considered for electrophysiological studies. Conduit punctures are usually performed with radiofrequency transseptal needles and PTA balloons. ${ }^{6,10}$ The electrophysiological catheters are placed via 3 pathways; transaortic approach for ventricular access, esophageal approach and trans-conduit puncture for atrial access.

It is challenging to puncture the Fontan conduit because of its unusual anatomy and presence of fibrosis around the conduit. In our study, the problem of unusual anatomy was overcome using cardiac computed tomography (CT) and intracardiac echocardiography. The cardiac and vascular anatomy was observed in detail and the conduit puncture site was planned using pre-procedure cardiac CT. Additionally, real-time intracardiac echocardiography was very useful in deciding where to puncture the conduit during the procedure. Radiofrequency transseptal needle was helpful in puncturing the fibrotic tissue around the conduit. However, the transseptal needle has a tendency to slide up along the conduit wall instead of puncturing it as the conduit wall is vertical. This was overcome by holding the dilator tip of the Swartz introducer with a snare catheter while using a transseptal needle. ${ }^{11}$

Puncture of the Fontan conduit made of Gore-tex is very challenging and time-consuming mainly because Gore-tex hardens over time. Planning an electrophysiological study in patients with Fontan conduit made of Gore-tex involves thorough preparation. This includes preparing for deep sedation or general anesthesia, placement of a Foley catheter, availability of various sizes and kinds of PTA balloons and dilators of an Inoue balloon.

We recommend the following steps in performing Fontan conduit puncture: (1) Fontan conduit angiography, (2) puncture with a Bronckenbrough transseptal needle stabilized by a snare, (3) puncture with a radiofrequency transseptal needle while holding it with a snare if that fails, (4) if success to puncture, but fail to insert the introducer into the atrium, inserting a wire into the atrium, (5) dilation with a PTA balloon, (6) if fail, dilation 
with a non-compliant balloon or a cutting balloon, (7) if fail, dilation with a dilator of an Inoue balloon. All steps should be guided by fluoroscopy and intracardiac echocardiography. Percutaneous trans-hepatic approach can be another option in case conduit puncture fails by all methods described above.

Electrophysiological studies in patients with congenital heart disease require the operator to be guided by various imaging studies and be thoroughly aware of the underlying cardiac and vascular anatomy. This is because every patient with congenital heart disease has a unique heart structure, irrespective of the similarities in the conditions. Additionally, general ECG criteria for tachycardia localization cannot be directly applied to these patients. The operator should also review and understand the previous cardiac surgery and intervention. A meticulous plan must be made for the procedure with particular attention paid to the types of electrophysiological catheters to be used, access routes for catheter positioning and appropriate X-ray beam angles for better visualization. A Three-dimensional (3-D) electroanatomical mapping system using multi-electrode mapping catheters is essential. ${ }^{12}$ Intracardiac echocardiography can be very useful for real-time visualization. The most important element in these procedures is to keep a 'never-give-up' spirit.

\section{Conclusions}

Conduit puncture is feasible and safe in patients with lateral tunnel and extracardiac Fontan circulation. Puncture of the Goretex conduit is more difficult and time-consuming than puncture of the pericardium conduit.

\section{Acknowledgements}

The authors thank Ji Wan Lyu, a radiologic technician in Severance Cardiovascular Hospital for his technical support.

\section{References}

1) Mackie AS, Ionescu-Ittu R, Therrien J, Pilote L, Abrahamowicz M, Marelli AJ. Children and adults with congenital heart disease los to follow-up; who and when? Circulation. 2009;120:302-309.
2) Fontan F, Baudet E. Surgical repair of tricuspid atresia. Thorax. 1971;26:240-248.

3) Laks H, Milliken JC, Perloff JK, Hellenbrand WE, George BL, Chin A, Di Sessa TG, Williams RG. Experience with the Fontan procedure. J Thorac Cardiovasc Surg. 1984;88:939-951.

4) AboulHosn JA, Shavelle DM, Castellon Y, Criley JM, Plunkett M, Pelikan P, Dinh H, Child JS. Fontan operation and the single ventricle. Congenital Heart Dis. 2007;2:2-11.

5) Chun KH, Uhm JS, Lee SE, Seo J, Yang PS, Choi JH, Kim NK. Transvenous implantation of a DDDR pacemaker in a patient with extracardiac conduit Fontan circulation. Korean J Med. 2015;88:299-302.

6) Dave AS, Aboulhosn J, Child JS, Shivkumar K. Transconduit puncture for catheter ablation of atrial tachycardia in a patient with extracardiac Fontan palliation. Heart Rhythm. 2010;7:413-416.

7) Nehgme R, Carboni MP, Care J, Murphy JD. Transthoracic percutaneous access for electroanatomic mapping and catheter ablation of atrial tachycardia in patients with a lateral tunnel Fontan. Heart Rhythm. 2006;3:37-43.

8) Khairy P, Fournier A, Ruest P, Vobecky SJ. Transcatheter ablation via a sternotomy approach as a hybrid procedure in a univentricular heart. Pacing Clin Electrophysiol. 2008;31:639-640.

9) Brown SC, Boshoff DE, Rega F, Eyskens B, Budts W, Heidbuechel H, Meyns B, Gewillig M. Transapical left ventricular access for difficult to reach interventional targets in the left heart. Catheter Cardiovasc Interv. 2009;74:137-142.

10) Correa R, Walsh EP, Alexander ME, Mah DY, Cecchin F, Abrams DJ, Triedman JK. Transbaffle mapping and ablation for atrial tachycardia after Mustard, Senning, or Fontan operation. J Am Heart Assoc. 2013;2:e000325.

11) Aoki $H$, Nakamura $Y$, Takeno $S$, Takemura T. A new procedure for a trans-conduit puncture by grasping the dilator tip with a snare catheter: an alternative access method during catheter ablation of supraventricular tachycardias after an extracardiac Fontan operation. Heart Rhythm. 2014;11:1492-1494.

12) Uhm JS, Kim NK, Lee H, Kim TH, Joung B, Pak HN, Lee MH. Usefulness of a crista catheter for 3-dimensional electroanatomical mapping of complex right atrial tachyarrhythmias. J Interv Card Electrophysiol.2015. Epub ahead of print. 